\title{
Modeling the weight of a grab bucket for digging
}

\author{
Ilya Tsipurskiy ${ }^{*}$ \\ Moscow State University of Civil Engineering, Yaroslavskoe shosse, 26, Moscow, 129337, Russia
}

\begin{abstract}
Modern construction of facilities of various types is accompanied by huge volumes of excavation works. The construction of surface and subsurface facilities requires the solution of a number of complex geotechnical problems, including those related to the creation of a deep underground cavity and a volume of hundreds of thousands of cubic meters. For soils of different categories of complexity of reservoir management at different depths, in some cases up to 50 meters, rope grab buckets are used. The article is devoted to the possibility of preliminary determination of the weight of an excavator grab bucket for excavation in developing the environment by creating various types of facilities. To determine the weight of the bucket, the existing empirical dependencies and the modeling method are used, which allows choosing the optimal weight of the excavator grab bucket for specific soil conditions. In this case, unlike the existing methods, the analytical calculations conducted do not only use the mass function of the soil developed, but also take into account the specificity of its resistivity to digging. Analytical dependence is given to determine the weight of the grab bucket for construction and practical recommendation of its preliminary choice for specific excavation works.
\end{abstract}

\section{Introduction}

In modern construction, excavation works play a large part that are production processes for the erection of earthwork structures and extraction of minerals. The labor intensity of excavation works causes the need for their maximum mechanization, massive scale and variety - the multiplicity of the nomenclature of the population of digging machines [4]. In relation to the method of erecting geotechnics, subsurface facilities erected with the opening of the earth's surface (or open type) are usually distinguished - basins, mines, trenches and without it (tunnels, subways, etc.) [5]. The construction of open-type earthwork structures is accompanied by the solution of a number of complex geotechnical problems, including those associated with the creation of a deep underground cavity and hundreds of thousands of cubic meters [7]. The construction of subsurface facilities in basins implies the erection of subsurface facilities in a previously open basin with backfilling it with soil and restoration of engineering landscaping. All these types of excavation works in the creation of underground space require specialized technology [8].

\footnotetext{
*Corresponding author: cil@bk.ru
} 
One of the types of this technique, used for digging soils of a different categories of complexity of reservoir management at different depths, in some cases up to 50 meters are excavators with a grab bucket. Grab buckets are also used for underwater mining of building materials and minerals. Ships equipped with a crane with a grab bucket are used for extraction of sand, gravel, ore from the bottom of rivers, lakes, seas and oceans. As a rule, a two-leaf grab bucket is used at the mechanical rope construction excavators and a lattice dragline boom, on the head of which blocks 9 and 10 are installed is used (Fig.).

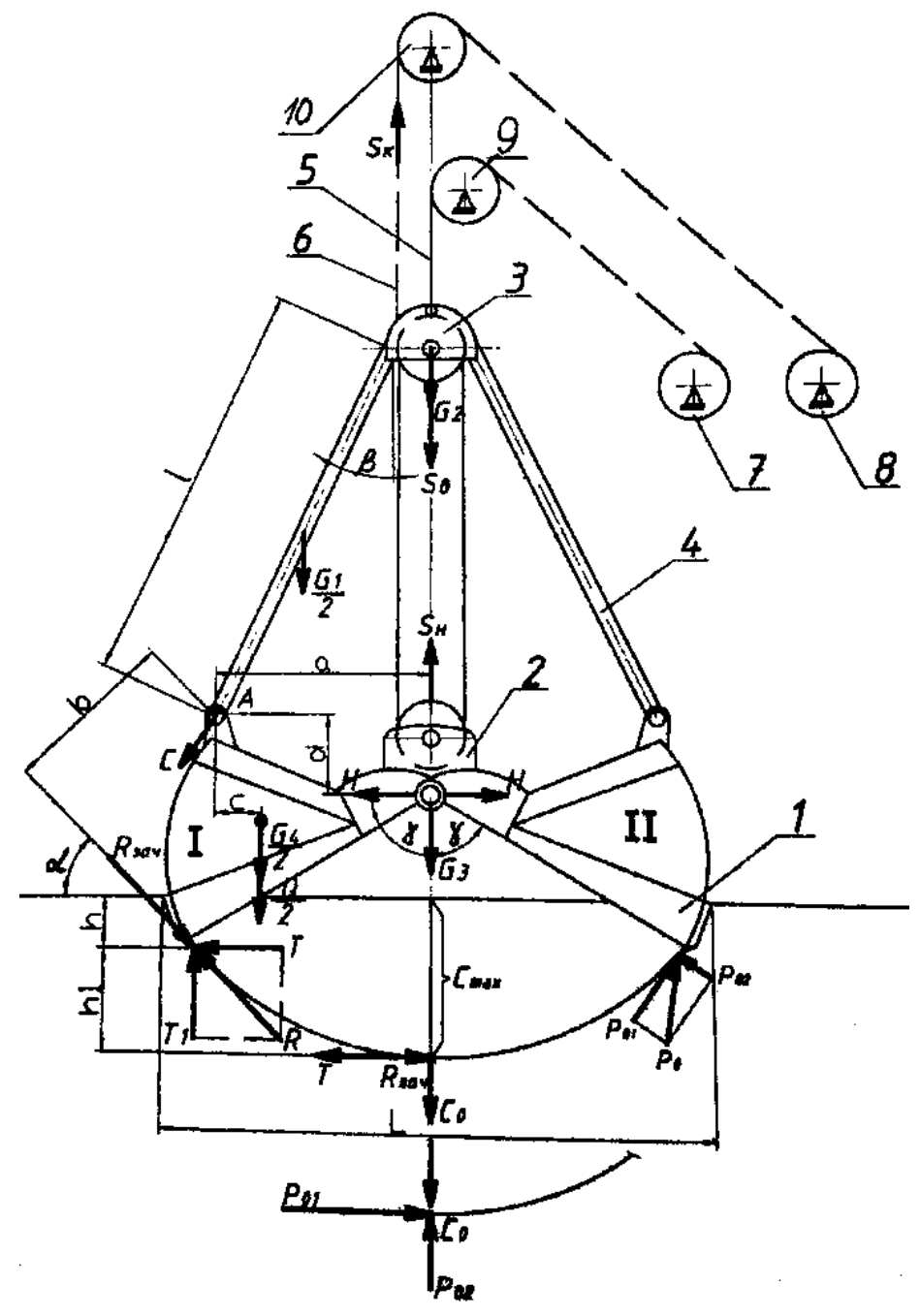

Fig.1. The device and pattern of forces acting on the grab bucket

The two-leaf grab bucket has crescent-shaped jaws I and II, which are pivotally connected to a lower crossbeam 2 and by means of rods 4 with a head 3 . A lifting rope 5, which is fixed to the head 3 , is intended for lifting and lowering the grab with the help of a lifting winch 7 . The closing rope 6 connected to the lower crossbeam 2 that passes through the hole in the head 3 and, bending around the block 10, is fixed to the drum of the closing winch 8. This rope is designed to open and close the jaws of the grab. To increase the digging efforts on the edges of the jaws, the closing rope is reeved in a pulley block, the clips of which are fixed to the lower crossbeam 2 and head 3 with the blocks. The winches 
have independent control. In the open position, the grab is lowered directly to the soil by releasing the supporting and closing ropes from the winch head. Closing of the grab is carried out by tightening the closing edge, as a result of which the jaws of the grab are closed, digging the material being loaded or removing the soil chip from the bottomhole. The grab is lifted in the case of simultaneous access of the supporting and closing ropes in the direction of ascent. The opening of the grab jaws occurs with the fixed supporting rope and the release of the closing rope. In this case, the jaws open under gravity and under the weight of the overloaded soil in the bucket (chip). Each jaw of the bucket is pivotally connected to the lower and upper crossbeams by drafts (supporting bases). Binding of the crossbeams with each other leads to the closing of the jaws, and the removal - to the opening. The lower crossbeam of the grab is movable. The management of the grab bucket consists of two actions: moving the bucket itself and manipulating its jaws. Depending on the kinematics of control, two types of grabs are distinguished: rope and drive (motor). Grabs used for digging are equipped with teeth 1 .

\section{Materials and Methods}

Referring to grabs, there are two main types in the technical literature that are fundamentally different in purpose. The first type includes grab buckets, the main purpose of which is soil digging. The second one - clamshells used for handling operations. One of the reasons for this is the difference in the processes occurring when digging loose material and digging soil.

In handling the bulk cargo, the entire scooped material is actively involved in scooping and the processes occurring in the loose medium when scooping are not substantially linear. The soil mechanics and mathematical model of the plastic flow of a loose medium have been developed in sufficient detail: the loose medium acts like an ideally plastic flow with a creep stress that depends on the average hydrostatic stress. It is this hypothesis that underlies the solution of a number of problems related to the interaction of indentors with the loose medium.

The destruction of cohesive soil by the grab working body occurs in the state of limit equilibrium of the soil, i.e. in a state immediately preceding the shift. The shear resistance $\tau$ for a connected medium with origin cohesion $c_{0}$ is represented as the following linear dependence (in terms of stress circle):

$$
\tau=c_{0}+\sigma \operatorname{tg} \rho,
$$

where $\sigma$-normal stress on a sliding surface;

$\rho$ - angle of friction or code of internal soil friction.

An environment where there is no origin cohesion $\left(C_{0}=0\right)$ is called ideal-granular; the medium in which there is no friction between the particles $\left(\rho=0 ; C_{0} \neq 0\right)$ is called ideallyconnected. For a medium with large connectivity, which is a particular case of a plastic medium, there is a functional dependence $\tau=f(\sigma)$, which can be equal to a certain constant value for an ideally connected medium. These well-known positions of ultimate equilibrium of any medium show that its resistance to destruction depends to a large extent on the friction value. Therefore, it is obvious that digging a stiff soil with a bucket of a grab requires a lot of effort and unit costs than loose material scooping. Local resistance $P i$, arising when scooping loose material, can be conditionally replaced by the common resistance $R$, applied to the edge of the jaw. The required value of the force of incision of the jaw into the bulk material $R_{\text {dig }}$, to overcome resistance $R$, can be determined using the experimental data [2], using the formula:

$$
R_{d i g}=\omega F, \kappa N
$$


where $\omega$-material resistance to digging, $\mathrm{\kappa Pa}$;

$F$ - cross-band square of material digging, $\mathrm{m}^{2}$.

The maximum value of this section occurs at the moment of complete closure of the jaws, when the horizontal component $T_{1}$ (see Fig., I - the left jaw: scooping process) coincides with the scooping force $R_{d i g}$ along the direction (but in different directions) and magnitude, and the vertical $T_{l}$ turns to zero according to this method of calculation.

When working in cohesive soils with a large drag, that is, when separating from a chip array by digging, the static loads on the cutting edge of the grab bucket represent the sum of resistance to cutting $-P_{c}$, friction $-P_{f}$ and the movement of the prism of dragging $-P_{p}$. Using the concept of soil resistivity to digging $K_{l}$, the value of the tangential component of resistivity at the cutting edge of the grab bucket $P_{01}$ is determined by the formula of Professor N.G. Dombrovsky [3]:

$$
P_{01}=K_{l} b C, \kappa N
$$

where $b$ - chip width; $C$ - chip thickness, removed from the bottomhole, $\mathrm{m}$.

Normal component to the same path of soil resistance to digging $P_{02}$ (soil pressure) is determined from the dependence:

$$
P_{02}=\psi P_{01}, \kappa N
$$

where $\psi$ - factor of proportionality.

The active value of this component largely depends on the weight of the digging grab bucket, which is a its mass function.

\section{Results}

The simplified dependence to determine the mass of the scooping grab bucket $\left(m_{g r}\right)$ as a function of its capacity $(q)$, the coefficient $\left(K_{f r}\right)$, taking into account the degree of difficulty of the works made by the grab and the density of the material being loaded $(\gamma)$ is: $m_{g r}=K_{f r} \cdot q \cdot \gamma$, that allows to set its weight $\left(G_{3}\right)$. The relationship between the intrinsic weight and the capacity of the scooping grab bucket is expressed by equations of different orders [6], in particular by the relation: $G_{3}=K_{m} \cdot q+0.5$, where: $K_{m}$ is a coefficient equal to a light grab-type 0.8 , for medium-1.5, for heavy-2.3 and in relation to the very heavy type this coefficient is equal to-3.0. In these relations, it is a question of scooping loose material and there is no account of the circumstances associated with the separation of soil chip from the subsoil.

With the flexible suspension of the grab on the ropes, the introduction of jaws into the soil bottomhole, removal of the chips and further filling the bucket during their binding is carried out largely under action of gravity of the grab. Therefore, the optimal choice of the weight of the grab with the specificity of taking into account the soil resistivity to digging $K_{l}$, is particularly important.

To establish the weight of the grab bucket with various capacities when a construction excavator is working on soils of different development difficulties, a modeling method was used to simulate the parameters of the grab bucket and, in particular, its weight. It was determined earlier [9] that, depending on the weight of the grab bucket installed on the construction excavator, it can develop soils of different difficulty categories. Therefore, a number of twelve grab buckets with capacity from 0.5 to 6.0 cubic meters were initially selected and divided into four groups with an interval of $0.5 \mathrm{~m}^{3}$. The composition of the buckets of each group hypothetically able to develop soils with the appropriate soil resistivity to digging $-K_{l}, \mathrm{KPa}$ is given in the table. 
Table 1. Estimated weight of buckets for different soils

\begin{tabular}{|c|c|c|c|c|c|}
\hline \multirow[b]{2}{*}{$\begin{array}{l}\text { Group } \\
\text { number }\end{array}$} & \multicolumn{5}{|c|}{ Parameters } \\
\hline & $\begin{array}{l}\text { Bucket capacity, } \\
\qquad q, \mathrm{~m}^{3}\end{array}$ & $\begin{array}{c}\text { Generalized } \\
\text { coefficient } K_{o}\end{array}$ & $\begin{array}{c}\text { Coefficient } K_{I}, \\
\mathrm{kPa}\end{array}$ & $\begin{array}{l}\text { Consistency of } \\
\text { soil } \gamma, \mathrm{t} / \mathrm{m}^{3}\end{array}$ & $\begin{array}{l}\text { Bucket } \\
\text { weight } \\
\boldsymbol{G}, \mathrm{kN}\end{array}$ \\
\hline \multirow{3}{*}{$\begin{array}{c}\text { First } \\
K_{\kappa}=1.0\end{array}$} & 0.5 & 50 & \multirow{3}{*}{$30 \div 120$} & \multirow{3}{*}{$\begin{array}{c}1.2 \div 1.5 \\
(1.35)\end{array}$} & \multirow{3}{*}{2.7} \\
\hline & 1.0 & 100 & & & \\
\hline & 1.5 & 150 & & & \\
\hline \multirow{3}{*}{$\begin{array}{c}\text { Second } \\
K_{K}=1.08\end{array}$} & 2.0 & 200 & \multirow{3}{*}{$120 \div 200$} & \multirow{3}{*}{$\begin{array}{c}1.4 \div 1.9 \\
(1.65)\end{array}$} & \multirow{3}{*}{10.5} \\
\hline & 2.5 & 250 & & & \\
\hline & 3.0 & 300 & & & \\
\hline \multirow{3}{*}{$\begin{array}{c}\text { Third } \\
K_{\kappa}=1.15\end{array}$} & 3.5 & 350 & \multirow{3}{*}{$180 \div 300$} & \multirow{3}{*}{$\begin{array}{l}1.6 \div 2.0 \\
(1.8)\end{array}$} & \multirow{3}{*}{20.7} \\
\hline & 4.0 & 400 & & & \\
\hline & 4.5 & 450 & & & \\
\hline \multirow{3}{*}{$\begin{array}{c}\text { Fourth } \\
K_{\kappa}=1.21\end{array}$} & 5.0 & 500 & \multirow{3}{*}{$250 \div 420$} & \multirow{3}{*}{$\begin{array}{c}1.9 \div 2.2 \\
(2.05)\end{array}$} & \multirow{3}{*}{33.6} \\
\hline & 5.5 & 550 & & & \\
\hline & 6.0 & 600 & & & \\
\hline
\end{tabular}

\section{Discussions}

In studies, the two-leaf grab bucket with a capacity of $q_{m}=0.01 \mathrm{~m}^{3}$ (10 liters) of $G_{L}=0.126, \mathrm{\kappa N}$ for digging in the soil channel was made as a laboratory model on empirical dependencies as recommended in the technical literature [10]. This laboratory bucket was the basic model during the formation of originals, corresponding final values of natural buckets, which are called integral analogs. The determination of the similarity coefficients $K_{L}$ (of linear scale) to establish the weight of each of the twelve buckets (hypothetically natural) with the corresponding capacity $q_{n}$ was carried out according to the following:

$$
K_{L}=\sqrt[3]{q_{n} / q_{m}}
$$

The weight of the bucket $G$ for a hypothetical series (the table shows only one weight value of a bucket of average capacity per group) was determined by the similarity theory using the generalized similarity (modeling) coefficient $K_{o}$. The generalized coefficient is a power-law dependence of the linear coefficient $K_{L}$. For linear, angular and specific parameters, the degree value is 1-one; for drag areas and forces the value of the degree is 2two, and for volume, weight and energy indicators, the degree value is 3-three [1].

At the beginning of digging the bucket model in the laboratory, the introduction of jaws into the soil occurs only under the weight of the grab. Then the soil response $P_{0}$ (see Fig., II - the right jaw: digging process) acting on the cutting edge is directed vertically and is decomposed into tangent $P_{01}$ and normal $P_{02}$ to the trajectory of the bucket edge making the soil resistance to digging. The ratio of these components $\psi=P_{02} / P_{01}$ during the movement of the bucket edge along the trajectory of digging varies from 0.2 to 0.5 [9]. Taking into account the available data on the ratio $\psi$ when digging only by turning the backhoe bucket of a hydraulic excavator and the existing analogy for conditions of the confirmatory analysis, it is recommended to take the ratio $P_{02} / P_{01}=0.5$ at the moment of jaw closure at point $C_{0}$.

In Fig. the point $C_{0}$ (the moment when the jaws are closed) with a short jaw trajectory element during digging is conditionally demolished downwards for convenience's sake to graphically show the ratio $P_{02} / P_{01}$. In general, the value of $P_{02}$ at this moment is determined by the response of soil to the jaw and is equal to the sum of half the weight of the grab 
$(G / 2)$ and the weight of soil in this jaw volume $\left(G_{d} / 2\right)$. We have $P_{01}=2 P_{02}$ from the previous equality and get:

$$
P_{01}=G+G_{2}, \kappa N
$$

The weight of the laboratory bucket $G_{L}$ is known, and the weight of the $G_{2}$ soil is set on a scale in a laboratory bucket. The coefficient of soil resistivity in the drip to digging by a laboratory bucket, which is equal to $K_{I L}=15$ on average, $к \mathrm{~Pa}$ is calculated by the value of the tangential component $P_{01}$ and the parameters of the maximal chips to be presented. From the ratio $\psi_{l}=K_{l} / K_{l L}$ (the values of soil resistivity to digging $K_{l}$, кPa by the natural bucket [3] are taken as average for each bucket in each of the four groups) the partial linear coefficient of similarity $-K_{\psi l}$ is determined, which is a component in the formation of the final values of the weight of natural buckets using the generalized coefficient $K_{o}$. This component is a partial reflection of the interconnection of soil density and its resistance to digging. When modeling the weight $G$ of natural digging buckets, their mass $m_{\kappa}$ was set by the bucket capacity $q$ and the average soil density $\gamma$ for this category of soil development difficulties. In this case, the experimental coefficient $K_{c}$, which characterizes the constructive mass of the grab bucket, was used [2].

\section{Conclusions}

As a result of modelling of the weight of the digging grab bucket, the physical properties of the soil (density) and its mechanical characteristics were used as arguments (the specificity of the soil resistivity to digging was taken into account). The conducted researches allowed to propose a calculation formula to preliminary determine the weight of the natural bucket of a construction excavator:

$$
G=0.1 \cdot q \cdot \gamma \cdot K_{\kappa} \cdot K_{o} / K_{\psi 1}, \kappa N
$$

It should be noted that the geography of studying issues related to grab equipment is enormous. There are many recommendations for determining the weight of the grab bucket as a function of its capacity in analytical form and in the form of curvilinear graphs [3,6]. The presented formula uses not only the mass function of the developed soil, but also takes into account the specificity of its resistivity to digging. Calculations carried out according to the formula (7) showed that the preliminary weight of the construction grab bucket, measured in $M N$, can be taken as $0.5 \div 0.6$ capacity for modeling excavation of soil with average values from recommended soil resistivities [3] bucket.

\section{References}

1. V.I. Balovnev, Similarity and modeling in the system of designing road-building machines (M .: MADI, 148, 2014)

2. A.A.Vainson, Hoisting-and-transport machines (Moscow, Mechanical Engineering, 430, 1975)

3. N.G. Dombrovsky, M.I.Galperin Baumaschine (Moscow,Hohschule, 224, 1985)

4. P.P. Ipatov, E.Yu. Pasechnik, Machinery and equipment for environmental management and water use (Tomsk: Publishing house of Tomsk Polytechnic University, 296, 2012)

5. D.S.Konyukhov, Construction of urban underground structures (Uch. Allowance, Moscow, Publishing House Architecture-S, 304, 2005)

6. B.A. Tauber, Grapple mechanisms (M. Mechanical Engineering, 272, 1985) 
7. V.I.Telichenko Modern technologies of integrated development of underground space megacities ( Moscow textbook / Publisher ASV, 2010)

8. I.L. Tsipurskiy, Settings digging and recruitment earthmoving machines (Moscow textbook/Publisher ASV, 223, 2016)

9. I.L.Tsipurskiy, Excavator with the working equipment dragline and grapple ( Moscow, MGSU, 56, 2011)

10. I.L.Tsipurskiy, Grab bucket parameters (Moscow, J. «Mechanization of construction" No. 12, 3, 2017 ) 